\title{
Minimally Invasive Valve Repair in Bi-Leaflets Prolapse Mitral Regurgitation (Barlow's Disease)
}

\author{
Alessandro Barbone ${ }^{1}$, Alessio Basciu ${ }^{1}$, Alessandra Iaccarino ${ }^{1}$, Ginevra Droandi ${ }^{1}$, Giorgio \\ Romano $^{1}$, Ines Andriani ${ }^{1}$, Mauro Chiarito ${ }^{2}$, Andrea Fumero ${ }^{1}$, Enrico Citterio ${ }^{1}$, Giuseppe \\ Crescenzi $^{1}$, and Lucia Torracca ${ }^{1}$ \\ ${ }^{1}$ IRCCS Humanitas Clinical and Research Center \\ ${ }^{2}$ Humanitas University
}

July 27, 2020

\begin{abstract}
Background and Aim of the Study: Barlow's disease is characterized by excess myxomatous degenerative tissue, leaflets prolapse and/or billowing, chordal-elongation, and annular-dilation. We reviewed our experience in Minimally Invasive Mitral Valve Repair (MIMVR) techniques to treat this complex mitral pathology. Methods: Between 1999 and 2017 a group of 125 patients was identified as Barlow's disease undergoing MIMVR. The diagnosis of Barlow disease was based upon preoperative transesophageal-echocardiography (TEE) and confirmed by the surgeon's assessment during open-heart procedure. Operative technique and data were retrospectively collected along with intra-hospital and long-term follow-up. Results: Successful Mitral Repair was possible in $100 \%$ of cases (125 patents) within the first cross clamp. Most patients (118 - 94.4\%) were treated by the Edge to Edge (Alfieri-Stich), while $2(1.6 \%)$ where corrected by neochordae implant and $2(1.6 \%)$ by quadrangular resection. 58 patients $(47.1 \%)$ received complete semi-rigid ring, while 65 (52.9\%) incomplete flexible partial ring excluding the LAM. Concomitant procedures were additional cleft occlusion in 10 cases (8\%), tricuspid valve repair (ring implant or remodeling) in 29 cases $(23.2 \%)$, left atrial ablation for atrial fibrillation in 4 cases $(3.2 \%)$ and atrial septal repair in 4 (3.2\%). Operative mortality was $0 \%$. Average long-term follow-up was $85 \pm 62$ months, with a survival rate of $97.6 \%$, freedom from redo mitral surgery of $98.4 \%$ and freedom from $>2+$ recurrent mitral regurgitation of $94.5 \%$. No difference in outcome could be related to annuloplasty ring type. Conclusions: Mitral repair can be safely and successfully achieved though minimal-invasive approach, with optimal long-term results.
\end{abstract}

Title: Minimally Invasive Valve Repair in Bi-Leaflets Prolapse Mitral Regurgitation (Barlow's Disease)

Authors : Alessandro Barbone M.D. Ph.D. ${ }^{1}$, Alessio Basciu M.D. ${ }^{1}$, Alessandra IaccarinoM.D. ${ }^{1}$, Ginevra Droandi M.D. ${ }^{1}$, Giorgio Romano M.D. ${ }^{1}$, Ines Andriani M.D. ${ }^{1}$, Mauro Chiarito M.D. ${ }^{2}$, Andrea Fumero M.D. ${ }^{1}$, Enrico Citterio M.D. ${ }^{1}$, Giuseppe Crescenzi M.D. ${ }^{3}$, Lucia Torracca M.D. ${ }^{1}$

Form the: UO of Cardiac Surgery ${ }^{1}$, : IRCCS Humanitas Clinical and Research Center, Via A. Manzoni 56, 20089 Rozzano (MI);

Department of Biomedical Sciences ${ }^{2}$, Humanitas University, Via Rita Levi Montalcini, 4, 20090 Pieve Emanuele (MI);

UO of Cardiac Anesthesia ${ }^{3}$; IRCCS Humanitas Clinical and Research Center, Via A. Manzoni 56, 20089 Rozzano (MI) 


\title{
Short Title: Minimally Invasive Mitral Repair
}

Word Count: 3857

Corresponding Author: Alessandro Barbone M.D. Ph.D.,

Area Medici Ovest 1,

IRCCS Humanitas Clinical and Research Center

Via A. Manzoni 56, 20089 Rozzano (Mi).

Phone: +39 0282244602

Fax: +3902 82244691

email:alessandro.barbone@humanitas.it

No conflict of interest or outsource of founding needs to be disclosed.

The present manuscript was presented as abstract at the:

$31^{\text {st }}$ EACTS Meeting - 07-11 October 2017 - Vienna, Austria

\begin{abstract}
Background and Aim of the Study:

Barlow's disease is characterized by an excess myxomatous degenerative tissue, leaflets prolapse and/or billowing, chordal elongation, and annular dilation. We reviewed our experience in Minimally Invasive Mitral Valve Repair (MIMVR) techniques to treat this complex mitral pathology.

\section{Methods:}

Between 1999 and 2017 a group of 125 patients was identified as Barlow's disease undergoing MIMVR. The diagnosis of Barlow disease was based upon preoperative transesophageal-echocardiography (TEE) and confirmed by the surgeon's assessment during open-heart procedure. Operative technique and data were retrospectively collected along with intra-hospital and long-term follow-up.
\end{abstract}

\section{Results:}

Successful Mitral Repair was possible in $100 \%$ of cases (125 patents) within the first cross clamp. Most patients (118 pts - 94.4\%) were treated by the Edge to Edge (Alfieri-Stich), while 2 cases (1.6\%) where corrected by neochordae implant and 2 cases $(1.6 \%)$ by quadrangular resection. 58 patients (47.1\%) received a complete semi-rigid ring, while $65(52.9 \%)$ an incomplete flexible partial ring excluding the LAM. Concomitant procedures were additional cleft occlusion in 10 cases (8\%), tricuspid valve repair (ring implant or remodeling) in 29 cases $(23.2 \%)$, left atrial ablation for atrial fibrillation in 4 cases $(3.2 \%)$ and atrial septal repair in $4(3.2 \%)$. Operative mortality was $0 \%$. Average long-term follow-up was $85 \pm 62$ months, with a survival rate of $97.6 \%$, freedom from redo mitral surgery of $98.4 \%$ and freedom from $>2+$ recurrent mitral regurgitation of $94.5 \%$. No difference in outcome could be related to annuloplasty ring type.

\section{Conclusions:}

Mitral repair can be safely and successfully achieved though minimal invasive approach, with optimal longterm results. 


\section{INTRODUCTION}

Minimally invasive mitral valve repair (MIMVR) has been proven as a valid alternative to full sternotomy and has become the standard of care in many centers. Some reports in the literature have shown that MIMVR can be safely used also in the context of complex mitral pathologies such as bileaflet prolapse in Barlow's disease $^{1}$. Barlow disease is characterized by excessive myxomatous leaflet tissue, bileaflet prolapse, chordal elongation and severe annulus dilatation. Recent histopathologic analysis have shown distinct changes in Barlow disease and fibroelastic deficiency confirming that they should be considered two separate entities ${ }^{2}$. Patients with Barlow disease often require surgery in young age and the possibility to offer a minimally invasive approach and a durable repair is particularly attractive for this young population. Different surgical techniques have been proposed to repair these complex valves. The objective of our study was to describe the early and long-term results of MIMVR in a large series of patients with Barlow disease.

\section{MATHERIALS and METHODS}

\section{Study Population}

Between 1999 and 2017 a cohort of 125 patients was identified as having Barlow's disease and not requiring concomitant other cardiac procedures that could not be performed through a Minimal Invasive Surgery (MIS) approach (e.g., coronary bypass, ascending aorta, or aortic valve surgery). The diagnosis of Barlow disease was made during the preoperative transesophageal echocardiography (TEE) examination and confirmed by the surgeon's direct assessment of the valve during open heart procedure.

The degree of MR was classified as grade 0 (absent or trivial), 1 (mild), 2 (moderate), 3 (moderate-to-severe), and 4 (severe MR), according to published literature ${ }^{3}$.

Baseline demographics and preoperative characteristics of the 125 patients are summarized in Table 1. Patient with previous right thoracotomy were excluded from this approach. Male and female patients are equally represented in the study population. Mean age was $43 \pm 12$ years. Mean preoperative left ventricle ejection fraction was $61.2 \% \pm 7.4 \%$. All patients were operated electively

\section{Surgical Technique}

MIMVR technique has been previously described ${ }^{4}$. Shortly a small (5-7 cm in length) right anterolateral minithoracotomy is performed in the $3^{\text {rd }}$ or $4^{\text {th }}$ intercostal space; cardiopulmonary bypass $(\mathrm{CPB})$ is instituted via femoral arterial and venous cannulation. Endoscopic vision is facilitated by means of $0^{\circ}$ or $30^{\circ}$ camera along with a full HD system. The aorta is cross clamped either by a Chitwood clamp ${ }^{5}$ or by a Cignet malleable clamp (VITALITEC, 10 Cordage Park Circle, Suite 100/200 Plymouth, MA 02360 USA) through the thoracotomy, and myocardial protection is achieved by antegrade delivery of crystalloid Custodiol cardioplegia $(2 \mathrm{~L})^{6,7}$. The left atrium is then open though the interatrial groove, and a left atrial retractor is used to expose the MV. $\mathrm{CO}_{2}$ was actively insufflated inside the chest cavity throughout the surgical repair and intracardiac air removal achieved by means of active ventricular and aortic root suction. Few different surgical technique are used in our experience: ring annuloplasty, neochordae implant (Gore-Tex; WL Gore \& Associates Inc, Flagstaff, Ariz), leaflet resection with sliding annuloplasty ${ }^{8}$, and Edge-to-Edge ("Alfieri") technique 9 . At the end of cardiopulmonary bypass (CPB) TEE is performed to evaluate the presence of residual MR and to measure MV area and gradient. MV area is evaluate through a trans gastric short axis view by a planimetric method. A complete echocardiographic evaluation is performed once the patient gains physical autonomy, usually on $4^{\text {th }}$ post-operative day: no patients is considered for hospital discharge with a residual mitral regurgitation greater than $2+$ (mild to moderate).

\section{Follow-up}

Clinical long-term follow-up was $100 \%$ complete and consisted of mitral valve function evaluation accordingly to the latest echocardiographic examination available (yearly check-up either with at our institution or at 
the referring cardiologist), reoperation for MV disease or any other cardiac reoperation, and patient survival. Follow up data were collected by telephone contact with the patient or family members, or by contact with the family physician. In those cases, in which MR grade was reported to be between 2 categories, it was rounded up to the worse grade. The average follow time after surgery was $85 \pm 62$ months.

\section{Statistical Analysis}

Continuous variables are reported as mean \pm standard deviation, and categoric variables are reported as percentages of group totals throughout the article. In case outliers might significantly alter perception of the real outcome, for the sake of completeness median and moda are also provided. The Kaplan-Meier method was used to estimate survival rate, freedom from MV reoperation, and freedom from recurrent MR greater than grade 2; furthermore, a Kaplan-Meier curve was arranged accordingly the different kind of annuloplasty ring used during surgery and results were calculate on the composite endpoint of survival + recurrence of MR and reoperation among groups, were comparing by Mantel-Haenszel test. Data for patients lost to follow-up were censored at the time of the last contact. Excel program (Microsoft Corp, Redmond, Wash) and Stata version XI (StataCorp, College Station, Tex) were used for statistical and survival analyses.

\section{RESULTS}

\section{Operative Technique and Outcomes}

Intraoperative data and MV repair techniques are listed in Table 2. As previously anticipated, anterolateral mini-thoracotomy with femoral cannulation for CPB was the surgical approach in all 125 patients ${ }^{4}$.

Repair techniques consisted of neochordae implant with the loop technique in 2 patients. Resection of the PML and sliding plasty was performed in other 2 patients. The Alfieri (Edge to Edge) ${ }^{9}$ technique was applied in 118 patients (94.4\%). In 105 patients (84\%) the stich was placed in the central valve area, in 17 patients $(10.4 \%)$ in paracommissural position, depending on the mechanism of mitral insufficiency and the jet localization.

A total of 10 patients (8\%) also received a concomitant cleft plication and 1 patients the suture of leaflet perforation.

123 patients (98.4\%) received a ring annuloplasty. A total of 58 patients (47.1\%) received a complete semirigid ring (average size $36 \pm 2.6 \mathrm{~mm}$ ), while the remaining 65 patients $(52.9 \%)$ received an incomplete flexible partial ring excluding the LAM (average ring size was $33.9 \pm 2.1 \mathrm{~mm}$ ). A subgroup analysis was performed to understand if any of the two devices offered any advantage in term of either survival or freedom from complication; a Kaplan-Meyer projection is reporting that no difference was recorded (Fig 4).

A group of 29 patients (23.2\%) received concomitant tricuspid valve repair: 23 by ring annuloplasty (average ring size $27 \pm 3 \mathrm{~mm}), 3$ patients by Kay plasty and 3 patients a De Vega repair.

Non-valvular associated procedure consisted of 4 PFO/ASD closure, and 4 left atrial ablation for atrial fibrillation treatment.

Average cardiopulmonary bypass time was $103 \pm 29$ min while average cardiac arrest time was $72 \pm 21 \mathrm{~min}$.

In the overall experience only in 1 case was required a conversion to full sternotomy because of inadequate mitral valve exposition (patient with pectus excavatum ) (Table 3).

In another single case a second cross clamp and cardiac arrest was required to allow retroflexion of the left atrial appendage that appeared inappropriately introflexed after weaning from cardiopulmonary bypass; the reason of this inconvenience was not clear neither the possible clinical significance but we felt safer to reinstitute the native cardiac anatomy by a new cross clamp and cardioplegia time.

In 3 patients Intraortic Ballon Pump (IABP) was required to facilitate weaning from cardiopulmonary bypass. 
One patient underwent to coronary angiography immediately after surgery due to abnormal ST elevation: angiography showed diffuse coronary spasm treated medically with resolution of the angiographic picture and ECG modification. The subsequent postoperative course was uneventful.

Three patients required a surgical revision for bleeding in the immediate post-operative time and 5 suffered (4\%) a Low Output Syndrome (LOS) during the Intensive Care Unit stay. Respiratory failure requiring prolonged mechanical ventilation occurred in 2 patients.

Overall, average ICU length of stay was $41 \pm 36.2$ hours (Median 30 h, Moda 12 h) (Table 4).

A total of 19 patients (15.2\%) suffered of postoperative Atrial Fibrillation along the subsequent hospitalization throughout discharge and overall 2 patients required permanent pacemaker before hospital discharge. 2 patients suffered a pleuro-pericardial effusion requiring percutaneous drainage during the first hospital admission.

Average post-operative hospital stay to discharge either home or to rehabilitative institute was $7.6 \pm 4.9$ days (Median and Moda 6 days).

Successful mitral repair at discharge was obtained in 125 patients (100\%), neither any patients requiring a mitral valve replacement nor any patients was discharged with a mitral regurgitation $>$ mild-moderate (grade $>2$ ). No mortality was recorded in the perioperative period (30 days).

\section{Follow up}

Average follow up time after surgery was $85 \pm 62$ months (Table 4). At follow up 3 patients were found dead: one due to neoplasia more than 130 months after surgery; one patient succumbed of sepsis after being re-operated because of mitral valve endocarditis 39 months after initial uneventful surgery; and 1 young (42 years old) female patient was found dead in a park while exercising 2.4 months after surgery. Overall, at echocardiographic control 7 patients presented $>2+$ recurrent mitral regurgitation (greater than moderate) at an average follow up time of $138 \pm 49.3$ months; 1 patient was found with moderate mitral stenosis, 2 case of mild mitral stenosis and 1 case of mild stenosis associate to mild regurgitation Two patients underwent redo mitral surgery (mitral replacement) at 40 and 186 months for recurrent symptomatic mitral regurgitation. Kaplan-Meier for the mentioned outcome are available at Figure 1 to 3. As shown in Figure 4, there were no significant differences in terms of overall mortality, reoperation or recurrence of MR (composite end-point) according to the different kind of annuloplasty ring used during surgery.

\section{DISCUSSION}

MIMVR has become the standard of care in many centers. Data from the literature have shown clinical advantages in terms of less bleeding and transfusion, less surgical site infection and postoperative atrial fibrillation, faster recovery and improved cosmesis ${ }^{10-13}$. We have introduced this surgical approach in the late 1990 and progressively expanded his application. Since the beginning our experience has accounted of more than 400 patients treated with minimal invasive mitral surgery; we feel that a significant learning curve is required for this procedure, as it is for most complex cardiac procedure. In the first 4 years of the experience the procedure was limited to more than ideal patients for more than ideal procedure. After roughly 40 patients the technique appeared to be sufficiently reproducible and under control to be available on a routine basis. Today we can easily affirm that is the first choice for young patients with atrioventricular valves or atrial cardiac surgical procedure. Although we feel that this technique requires continuous application and practice to be safe and sound not only for the surgeon, but for the entire surgical team.

Barlow patients are usually a young population (our mean age was $43+11.9$ years) thus highly motivated to receive a minimally invasive procedure and obviously a durable repair. On the other hand, they have a complex valvular disease still considered a challenging procedure for mitral repair. Barlow disease is characterized by proteoglycans accumulation in the spongiosa layer and intimal thickening both contributing to leaflets thickening. Macroscopically Barlow valves show excess tissue, leaflets thickening, chordal elongation, 
bileaflet prolapse, annulus dilatation and, not rarely, annular calcification This extensive valve remodeling and the global involvement of all valve structures into the disease process, make the valve repair particularly demanding. Nevertheless, a variety of surgical techniques have been proposed to repair Barlow valves.

In the current study, neochordae implantation was used in 2 patients. In 2 other patients a quadrangular resection with sliding plasty of the posterior leaflet was performed accordingly to Carpentier technique ${ }^{8}$. Most of the patients ( $n=118: 94.4 \%)$ received a valve repair using the edge-to edge technique. As described by Alfieri's group, the technique is effective in restoring mitral competence in Barlow disease with good early and long-term results ${ }^{9}, 14$. One hundred and five patients (84.0\%) received a central edge-to-edge while 13 (10.4\%) a paracommissural stich, depending on the valve pathology and regurgitant jet localization.

In Barlow valves the technique of stich placement is a key point for the surgical result. The edge-to-edge stich should be placed deep in the body of the leaflets to decrease the height of the leaflets and move the coaptation point within the left ventricle, below the mitral annulus level. In patients with particularly high risk of SAM due to anatomical reason (basal septal hypertrophy, narrow mitral-aortic angle) the edge-to-edge stich can be placed, at level of the posterior leaflet, few millimeters far away from the posterior mitral anulus. This surgical trick shifts the coaptation point of the mitral valve toward the posterior mitral annulus, moving the anterior mitral leaflet away from the LV outflow tract and avoiding SAM. Indeed, in our study we didn't observed the occurrence of SAM in any of the 125 patients.

At 10 years follow up the expected survival according to the Kaplan Meier estimates is $99.2 \pm 1.6 \%$, while at 15 years is $96.3 \pm 5.8 \%$. Freedom from $M R>2+$ was $98.7 \% \pm 2.4 \%$ at 10 years and $77.8 \pm 19.2 \%$ at 15 years: a total of 7 patients were found with a moderate or more recurrent Mitral Regurgitation at follow up. Freedom from reoperation was $98.8 \% \pm 2.4 \%$ at 10 years, same rate at 15 years, accounting for a total of 2 reoperation in the overall follow up.

In the current study all but 2 patients (characterized by particular calcified and surgically unapproachable mitral annulus) undergoing MV repair received an annuloplasty ring: two different type of ring were used in our experience: either a complete semi-rigid annuloplasty ring in 58 cases $(47.1 \%)$ or incomplete flexible partial ring in 65 patients $(52.9 \%)$. The incomplete rings were used in an average smaller size compared to the complete $(33.9 \pm 2.1 \mathrm{~mm}$ vs $36 \pm 2.6 \mathrm{~mm})$ but this seemed do not affect neither the short nor the long-term outcome. Debate between surgical philosophies on which is the most reliable device for mitral annuloplasty has been ongoing for years ${ }^{15}$. The intention of restoring anatomically accurate mitral valve has driven Carpentier's development of a rigid prosthetic annuloplasty ring. The ring is intended to restore the normal systolic size and shape of the annulus, thus restoring leaflet coaptation and preventing further deformation in time of the annulus. The ring size is based on precise measurement of the anterior leaflet surface area, approximating the size and shape of the mitral orifice in systole ${ }^{8,16}$.

Although, thanks to further development of cardiac imaging technique, in particular 3D echocardiography, was elucidated the dynamic structure of the mitral annulus and its evolution along the cardiac cycle. Indeed, to allow a functional restoration of the mitral valve physiology, a flexible and incomplete annuloplasty ring sparing the anterior leaflet was developed and implemented to maximize the mitral annular excursions ${ }^{17,} 18$ and limit the diastolic restrain on the leaflets.

In our series this trend was followed, favoring the complete annuloplasty ring at the beginning of the surgical experience, moving to a more flexible and incomplete ring once surgical data was accumulating on this latter device. Notably in recent published revaluations of past surgical experience, partial rings showed lower rest and exercise trans mitral gradients, predicting a more favorable clinical outcome in terms of echocardiographic atrial remodeling (smaller left atrial size predicting lower atrial fibrillation rate) and functional performance (better 6 minutes walking test of Heart Failure symptoms tolerance) without affecting long term outcome of mitral repair ${ }^{19,} 20$.

It has to be noted how an incomplete ring sparing the LAM, further simplifies the minimal invasive approach reducing the number of suture required in an especially complex area to be exposed, rendering device preferable. 
Thus can be underlined how direct comparison of our finding with other contemporary series shows reproducible results.

Munaretto and colleagues are reporting 50 cases of MIS in Barlow disease over a 3 years timeframe, with an average follow up of 36 months with a successful repair through either resection approach (P2 resection and neocordae on LAM) or non-resection approach (neocordae on LPM and LAM) apparently without differences in late outcome ${ }^{21}$. The average cross clamp and cardiopulmonary bypass time is substantially longer compared to what reported in our and other different experience ${ }^{22}$, due most likely to the more complex repair approach. This apparently is not affecting neither the short nor the long term follow up, indeed allowing an overall shorter ICU and hospital stay.

Borger and colleagues reported their experience with MIMVR in Barlow Disease using different surgical techniques $^{22}$. All patients undergoing MV repair but 1 patients received a partial ring annuloplasty with a mean ring size of $35.7 \pm 2.8 \mathrm{~mm}$. Freedom from reoperation was $96.8 \%$ at 5 years, and $93.8 \%$ at 10 years. Late echocardiographic follow-up showed freedom from moderate-to-severe MR in $90,2 \%$ at 5 years and $88.4 \%$ at 11 years.

Our surgical results favorably compare also with large surgical series in which mitral repair has been performed via median sternotomy and long term follow up has been reported.

Flameng and colleagues reported a series of 348 patients with both Barlow and fibroelastic deficiency ${ }^{23}$. Freedom from recurrent MR $(>2+)$ was $82.2 \%$ at 5 years and $64.9 \%$ at 10 years for the entire group, with worse outcomes for patients with Barlow's disease.

David and colleagues are reporting $840 \mathrm{MV}$ repair in median sternotomy with very long follow up. At 20 years, freedom from recurrent severe MR was $90.7 \%$, and freedom from moderate or severe MR was $69.2 \%$ for the entire group ${ }^{24}$. Advanced mixomatous degeneration, like Barlow disease, as long as anterior mitral prolapse, is recognized as a predictor of valve failure.

De Bonis, from the Alfieri's group , reports on long term follow up of 174 patients treated by edge-to-edge technique through median sternotomy and comprising Barlow disease patient ${ }^{25}$. Freedom from MR $>3$ was $83,8 \%$ at 14 years with no difference between anterior and bileaflet mitral prolapse. Residual MR at discharge being the only predictors of MR recurrence.

A particularly interesting reports is from Zekry and colleagues that are proposing to treat MR in Barlow by a simple annuloplasty ring ${ }^{26}$. In their manuscript they are claiming that Patients with Barlow disease have enormously enlarged annulus size and extremely excessive leaflet tissue thus placement of a mitral annuloplasty ring remodels the MV shape and reduces annulus size. As the annulus size decreases, leaflets get closer and are pushed down toward the left ventricular apex. According to authors this technique not only bears a much shorter operative time, but even a better long-term outcome hitting a $100 \%$ freedom from recurrent MR. However, this approach limited to a simple annuloplasty without any tissue resection or rearrangement bares a couple of serious concerns, although it might highly simplify MIMVR. First, excessive tissue may cause functional MV stenosis, and second, it heightens the risk of postoperative SAM (as proven by the occurrence of this complication in 7 over 24 patients although apparently medically solved in most cases). Thus, in our center we are not keen to accept this pathophysiological approach to mitral valve repair.

The results of our study confirm that patients with Barlow disease can be safely treated with a minimally invasive procedure. The use of the edge to edge technique has provided very good long-term results favorably comparing with the data available in the literature.

Both surgical experience in mitral repair and minimally invasive surgery are mandatory to obtain good results and exposure to a large number of procedure is the precondition to hit this goal. 


\section{Study Limitations}

Our study is retrospective in nature and therefore subject to the inherent weaknesses of a retrospective analysis. First limit is related to the lack of comparison with a sternotomy group of patients treated during the same period by the same surgeons. Furthermore, echocardiographic follow up evaluation is not ideal not being performed in our echo lab but only obtained as a report from outside institution. Only data regarding the outcome of mitral valve repair are available and no other information neither on LV function nor dimension or mechanism of valve failure are available.

\section{Conclusions}

MIMVR can be safely used to treat mitral regurgitation in patients with complex Barlow disease. Long term follow up has shown good results in our quite large population of patients treated predominantly with edge-to-edge technique. Surgical experience on mitral repair and minimally invasive surgery are mandatory to obtain reproducible long-term results.

\section{Author Contribution to the research and Manuscript:}

Alessandro Barbone M.D. Ph.D.: Concept/design, Data analysis/interpretation, Drafting article, Statistics, Data collection

Alessio Basciu M.D.: Drafting article, Data collection

Alessandra IaccarinoM.D.: Data analysis/interpretation, Data collection,

Ginevra Droandi M.D.: Data collection

Giorgio Romano M.D.: Concept/design, Data collection

Ines Andriani M.D. Data collection

Mauro Chiarito M.D.: Data analysis/interpretation, Critical revision of article, Statistics

Andrea Fumero M.D.: Data analysis/interpretation, Approval of article,

Enrico Citterio M.D.: Data analysis/interpretation, Critical revision of article

Giuseppe Crescenzi M.D.: Concept/design, Data analysis/interpretation, Critical revision of article,

Lucia Torracca M.D.: Concept/design, Data analysis/interpretation, Drafting article

\section{References:}

1. Davierwala PM, Seeburger J, Pfannmueller B, Garbade J, Misfeld M, Borger MA and Mohr FW. Minimally invasive mitral valve surgery: "The Leipzig experience". Annals of Cardiothoracic Surgery . 2013;2:744-750.

2. Hjortnaes J, Keegan J, Bruneval P, Schwartz E, Schoen FJ, Carpentier A, Levine RA, Hagège A and Aikawa E. Comparative Histopathological Analysis of Mitral Valves in Barlow Disease and Fibroelastic Deficiency.Seminars in Thoracic and Cardiovascular Surgery . 2016;28:757-767.

3. Enriquez-Sarano M, Avierinos J-F, Messika-Zeitoun D, Detaint D, Capps M, Nkomo V, Scott C, Schaff HV and Tajik AJ. Quantitative Determinants of the Outcome of Asymptomatic Mitral Regurgitation. New England Journal of Medicine . 2005;352:875-883.

4. Lapenna E, Torracca L, De Bonis M, La Canna G, Crescenzi G and Alfieri O. Minimally Invasive Mitral Valve Repair in the Context of Barlow's Disease. The Annals of Thoracic Surgery . 2005;79:1496-1499.

5. Chitwood WR, Elbeery JR and Moran JF. Minimally invasive mitral valve repair using transthoracic aortic occlusion. The Annals of Thoracic Surgery . 1997;63:1477-1479. 
6. Savini C, Murana G, Di Eusanio M, Suarez SM, Jafrancesco G, Castrovinci S, Castelli A and Di Bartolomeo R. Safety of Single-Dose Histidine-Tryptophan-Ketoglutarate Cardioplegia During Minimally Invasive Mitral Valve Surgery. Innovations:Technology and Techniques in Cardiothoracic and Vascular Surgery . 2014;9:416420.

7. Edelman JJ, Seco M, Dunne B, Matzelle SJ, Murphy M, Joshi P, Yan TD, Wilson MK, Bannon PG, Vallely MP and Passage J. Custodiol for myocardial protection and preservation: a systematic review. Ann Cardiothorac Surg . 2013;2:717-28.

8. Carpentier A, Relland J, Deloche A, Fabiani JN, D'Allaines C, Blondeau P, Piwnica A, Chauvaud S and Dubost C. Conservative management of the prolapsed mitral valve. Ann Thorac Surg . 1978;26:294-302.

9. Maisano F, Schreuder JJ, Oppizzi M, Fiorani B, Fino C and Alfieri O. The double-orifice technique as a standardized approach to treat mitral regurgitation due to severe myxomatous disease: surgical technique.Eur J Cardiothorac Surg . 2000;17:201-5.

10. Citterio E, Manasse E, Pilato E, Eusebio A, Bandera A and Gallotti R. Port-access cardiac surgery: clinical experience with first 50 cases.Ital Heart $J$. 2001;2:904-9.

11. Torracca L, Lapenna E, De Bonis M, Kassem S, La Canna G, Crescenzi G, Castiglioni A, Grimaldi A and Alfieri O. Minimally invasive mitral valve repair as a routine approach in selected patients. J Cardiovasc Med (Hagerstown) . 2006;7:57-60.

12. Seeburger J, Borger MA, Falk V, Kuntze T, Czesla M, Walther T, Doll N and Mohr FW. Minimal invasive mitral valve repair for mitral regurgitation: results of 1339 consecutive patients. European Journal of Cardio-Thoracic Surgery . 2008;34:760-765.

13. Cheng DCH, Martin J, Lal A, Diegeler A, Folliguet TA, Nifong LW, Perier P, Raanani E, Smith JM, Seeburger J and Falk V. Minimally Invasive Versus Conventional Open Mitral Valve Surgery: A MetaAnalysis and Systematic Review. Innovations:Technology and Techniques in Cardiothoracic and Vascular Surgery . 2011;6:84-103.

14. Alfieri O, Maisano F, De Bonis M, Stefano PL, Torracca L, Oppizzi M and La Canna G. The doubleorifice technique in mitral valve repair: a simple solution for complex problems. J Thorac Cardiovasc Surg . 2001;122:674-81.

15. Schubert SA, Mehaffey JH, Charles EJ and Kron IL. Mitral Valve Repair: The French Correction Versus the American Correction.Surgical Clinics of North America . 2017;97:867-888.

16. Carpentier A. Cardiac valve surgery: The 'French correction'. The Journal of Thoracic and Cardiovascular Surgery . 1983; Volume 86:323-337.

17. Spratt JA. Non-resectional repair of myxomatous mitral valve disease: the 'American Correction'. J Heart Valve Dis . 2011;20:407-414.

18. Lawrie GM, Earle EA and Earle NR. Nonresectional Repair of the Barlow Mitral Valve: Importance of Dynamic Annular Evaluation. The Annals of Thoracic Surgery . 2009;88:1191-1196.

19. Chan KL, Chen S-Y, Chan V, Hay K, Mesana T and Lam BK. Functional Significance of Elevated Mitral Gradients After Repair for Degenerative Mitral Regurgitation. Circulation: Cardiovascular Imaging . 2013;6:1041-1047.

20. Mesana TG, Lam BK, Chan V, Chen K, Ruel M and Chan K. Clinical evaluation of functional mitral stenosis after mitral valve repair for degenerative disease: Potential affect on surgical strategy. The Journal of Thoracic and Cardiovascular Surgery . 2013;146:1418-1425.

21. Muneretto C, Bisleri G, Bagozzi L, Repossini A, Berlinghieri N and Chiari E. Results of minimally invasive, video-assisted mitral valve repair in advanced Barlow's disease with bileaflet prolapse. Eur J Cardiothorac Surg . 2015;47:46-50; discussion 50-1. 
22. Borger MA, Kaeding AF, Seeburger J, Melnitchouk S, Hoebartner M, Winkfein M, Misfeld M and Mohr FW. Minimally invasive mitral valve repair in Barlow's disease: Early and long-term results. The Journal of Thoracic and Cardiovascular Surgery . 2014;148:1379-1385.

23. Flameng W, Meuris B, Herijgers P and Herregods M-C. Durability of mitral valve repair in Barlow disease versus fibroelastic deficiency. The Journal of Thoracic and Cardiovascular Surgery . 2008;135:274-282.

24. David TE, Armstrong S, McCrindle BW and Manlhiot C. Late Outcomes of Mitral Valve Repair for Mitral Regurgitation Due to Degenerative Disease. Circulation . 2013;127:1485-1492.

25. De Bonis M, Lapenna E, Lorusso R, Buzzati N, Gelsomino S, Taramasso M, Vizzardi E and Alfieri O. Very long-term results (up to 17 years) with the double-orifice mitral valve repair combined with ring annuloplasty for degenerative mitral regurgitation. The Journal of Thoracic and Cardiovascular Surgery . 2012;144:1019-1026.

26. Ben Zekry S, Spiegelstein D, Sternik L, Lev I, Kogan A, Kuperstein R and Raanani E. Simple repair approach for mitral regurgitation in Barlow disease. The Journal of Thoracic and Cardiovascular Surgery . 2015;150:1071-1077.e1.

\section{Figure Legend}

Fig 1: Kaplan Meier Estimate Survival with Population at Risk

CI: 95\% Confidence Interval, MR: Mitral Regurgitation; Data Reported As Mean \pm SD

Fig 2: Kaplan Meier Estimate Survival Free of Recurrence of Mitral Regurgitation $>2+$

CI: 95\% Confidence Interval, MR: Mitral Regurgitation; Data Reported As Mean \pm SD

Fig 3: Kaplan Meier Estimate of Survival Free of Reoperation:

CI: 95\% Confidence Interval, Data Reported As Mean \pm SD

Fig 4: Kaplan Meier Estimate of survival in subgroups arranged by annuloplasty ring type.

CI: $95 \%$ Confidence Interval, Data Reported As Mean \pm SD

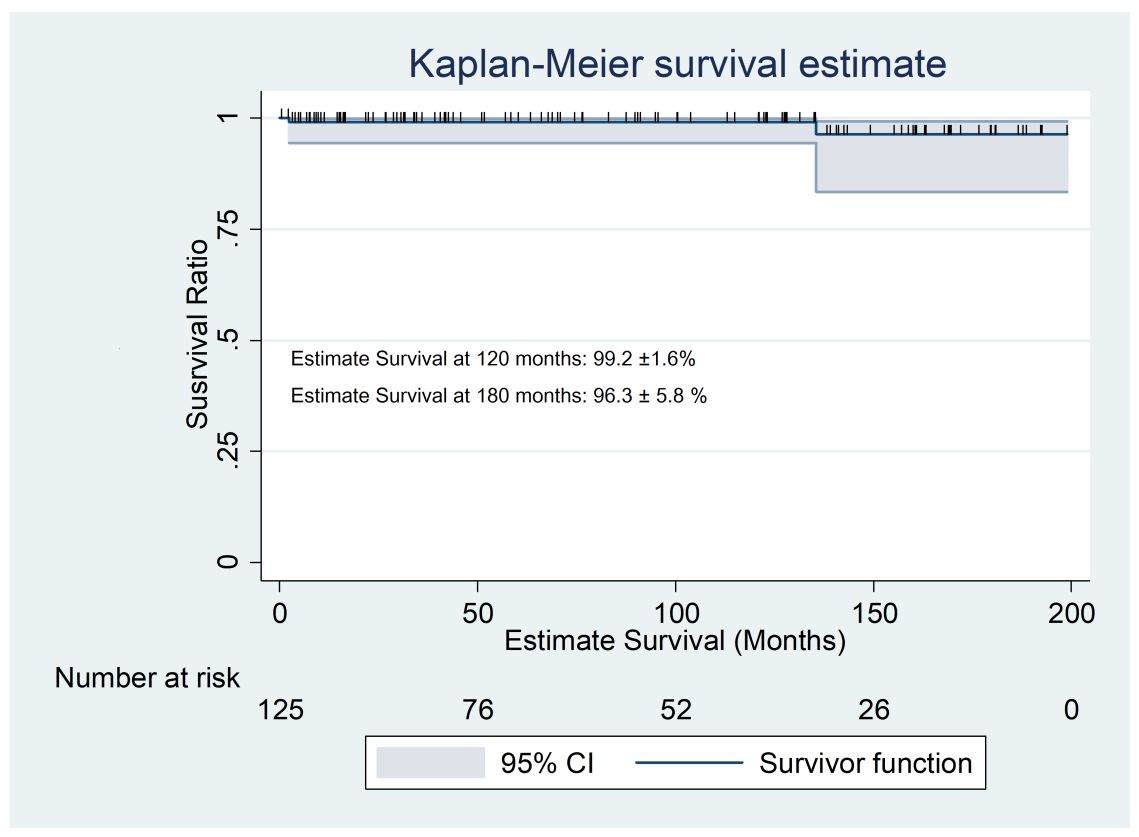



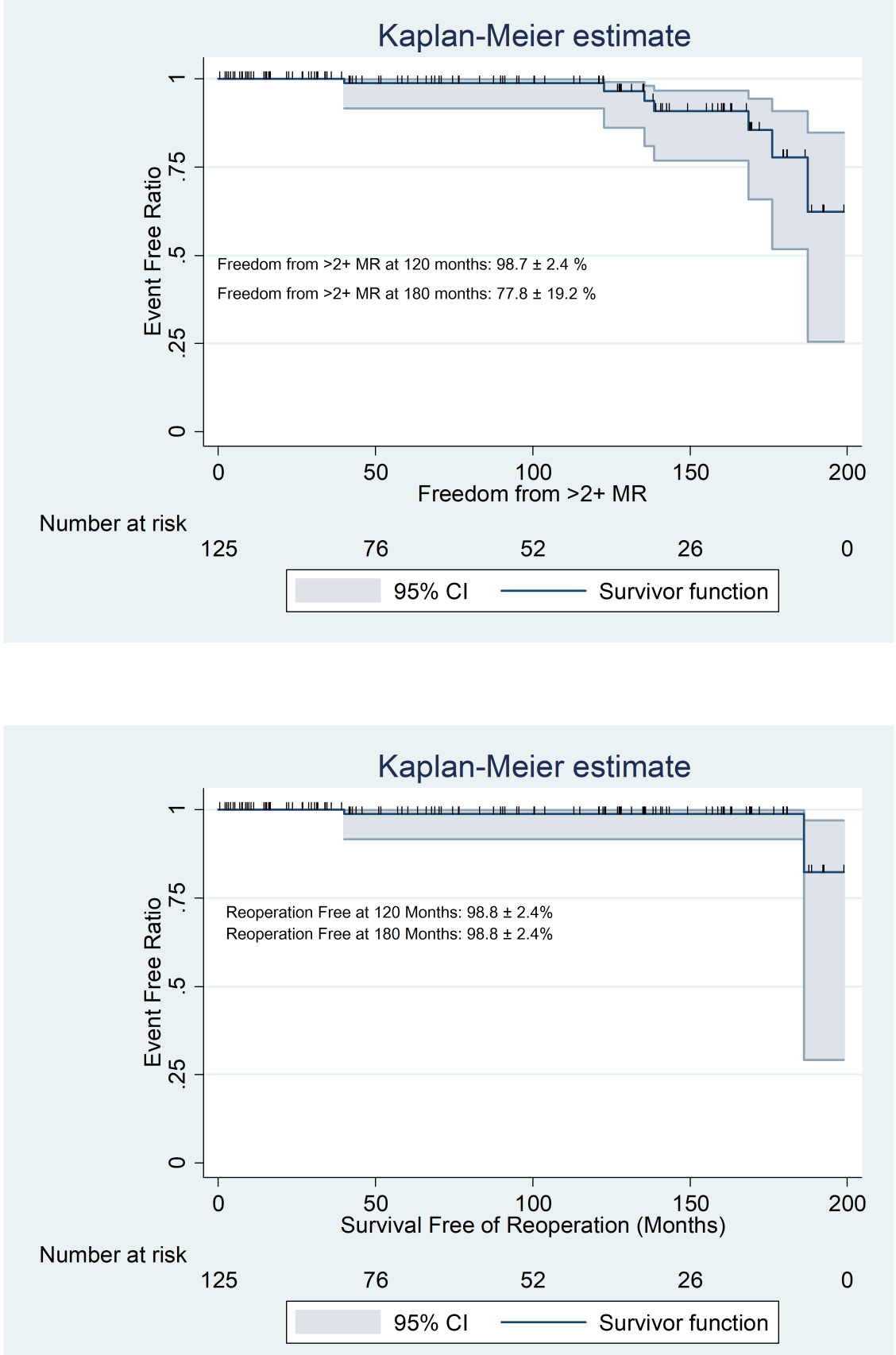


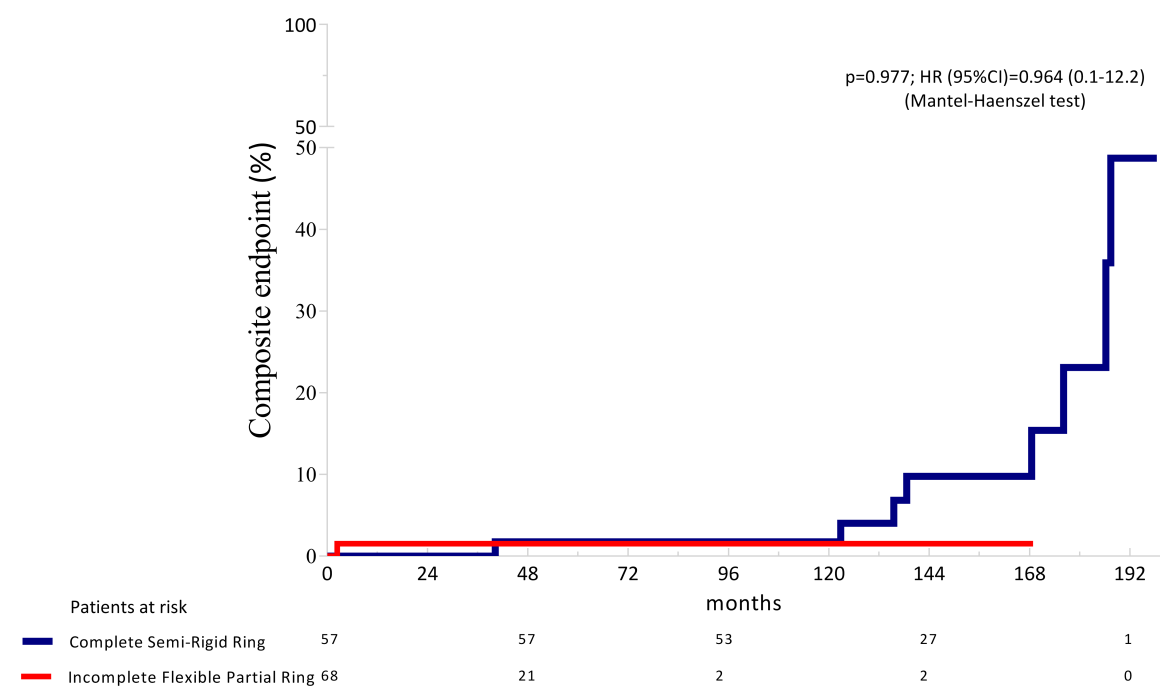

